\title{
Comparative Study of Alternative Computing Technologies for Speed and Density
}

\author{
Tarik Ozkul \\ Computer Engineering Division, American University of Sharjah, Sharjah, UAE
}

\begin{abstract}
As we design faster and denser silicon hardware to increase computing performance, some limitations started to appear. It is apparent that these limitations will stifle the growth of performance in the near future. In order to overcome these limitations, alternative computing technologies started to emerge. Among many attempts, biological computing, DNA computing, quantum computing and optical computing emerged as promising technologies. In this present paper we give state of the art of alternative computing technologies and compare them from the viewpoint of speed and density and classify the type of problems they can help to solve. The driving force behind the search for alternative technologies and the major trends are also explained.
\end{abstract}

Keywords: DNA computing, biological computing, whole cell computing, quantum computing, reversible computing, optical computing, high performance computing.

\section{Introduction}

In accordance with the Moors law, the density of IC chips keep increasing at a steady rate. So far the rule has worked very well and it seems that, using better photolithographic tools, we can keep reducing the size of the IC chips. As of 2002, some IC manufacturers have started using $193 \mathrm{~nm}$ ultraviolet light for deep-ultraviolet lithography to manufacture very dense microchips. Some manufacturers have been developing extreme ultraviolet lithography (EUV) which will utilize $13 \mathrm{~nm}$ light wavelength. With this technology it is expected to have the ability to print 40 atom wide transistors on silicon chip [1].

Even though it will be physically possible to pack more and more transistors on a VLSI pack- age, heat dissipation and energy consumption of the chip is likely to limit the number of transistors to pack in a chip. As more and more transistors/devices get packed on a chip, the power consumption and heat generated will increase. Even if more efficient technologies can be developed to reduce the power consumption and increase the packing density to 1017 logic devices in a cubic centimeter, theoretical power consumption of such a device will be enormously high $[2,3]$. According to the underlying physics, an ideal conventional device must dissipate at least $\ln (2) \times \mathrm{kT}$ joules in switching; so an ideal device packing 1017 transistors, operating at room temperature at a frequency of 10 gigahertz, would dissipate $>3,000,000$ watts:

$$
\begin{aligned}
& \ln (2) \times \mathrm{kT} \text { at } \mathrm{T}=300 \mathrm{Kelvin} \\
& 10^{17} \times 3 \times 10^{-21} \times 10 \times 10^{9}=3,000,000 \text { watts }
\end{aligned}
$$

Performances of the actual conventional devices seem to be much lower than the ideal theoretical figures given above [4]. With the current technology 55x106 transistor count Pentium IV chip operating at $2 \mathrm{Ghz}$ consumes about 60 watts of power. With this type of energy consumption rate - which is far from ideal - any device with 1017 transistor count will be consuming around $160,000,000$ watts. Obviously, with this type of energy consumption it makes no sense to increase the transistor count any more.

These requirements started pushing researchers toward alternative computing technologies which consumes much less power yet in can be very dense. In this paper we will be reviewing several alternative computing technologies 
which may change the shape of computing in the near future.

\section{Basic Requirements from Alternative Computing Technologies}

Traditional digital computing architecture uses logic gates for decision making. Extending the same concept to the alternative technologies, we can state the following are the requirements for any technology to be used for computing.

1) Should have a mechanism for decision making.

2) Should be able to pack large quantities of decision making devices in a small physical space,

3) Should have reasonable power consumption.

The following technologies satisfy the above conditions and seem promising as alternative computing technologies.

\section{Alternative Technologies}

Currently, there are three different technologies emerging as promising competitors to become the mainstream technology of the future computing, promising to meet the requirements of high density, high performance and low power consumption. All technologies are at their infancy and still much work needs to be done before the technology becomes mainstream production technology. The technologies and the class of problems they are suitable for are discussed below.

\subsection{Biocomputing}

Biocomputing is emerging as an alternative for high density, high performance computing of computationally difficult problems. Massive developments in this area indicate that biocomputing is indeed possible and may very well be manufactured like the silicon technology in the near future. A visionary talk by Richard Feynman in 1959 described the dream of constructing micro scale computers. It seems that the dream of Feynman is turning into reality day by day. By observing the nature carefully, we have noticed that biological systems are far more efficient and compact than anything that is humanly engineered. As an example, a single cell of Escherichia coli bacteria with a diameter of 1 micrometer has a digital storage capacity of a high density floppy disk. When we look at biological systems at nanoscale, it is possible to find analogues of all engineering tools that we use; programmable control systems, wires, shafts and even motors [5].

In order for any system to perform computing function it should have the ability to select a path exercising its decision. In a traditional computing device this function is performed by logic gates. Event though a logic gate performs a very simple function, collection of these simple gates can perform very complex tasks as computing machines. Biological sensing and computing using nucleic acids, enzymes and whole cells are successfully tried recently $[6,7]$. It is known that individual cells can perform very complex tasks such as searching for nutrients.

There are two major trends for biological computing. One of the trends uses DNA, which is the information storage element available in every cell, for computing purposes. The other trend uses biological cells as a whole to perform computational operations.

\section{DNA Computing}

DNA (deoxyribonucleic acid) is a genetic storage element available in every living cell. DNA computing relies on devising algorithms for solving equations using the encoded information stored in DNA. Computing through DNA makes use of the following facts [8].

A natural DNA strand is a succession of four different building blocks called bases ordered in a specific way. These bases are known as Adenine, Guanine, Thymine and Cytosine or as $A, G, T$ and $C$ in short. DNA is made up of sequences of these bases and the ordering is unique for every living organism. Currently it is possible to synthesize these bases synthetically in a test tube.

There exists a set of molecular biology tools to manipulate DNA strands. These tools are as follows:

1. Synthesizing: Building a desired length strand using the four different A,G,T and C bases. 
2. Mixing: Combining different size DNA strands in a test tube.

3. Annealing: Bonding together different DNA sequences.

4. Melting: Breaking double stranded DNA apart into single strands.

5. Copying: Making copies of DNA strands.

6. Separating: Separating the strands.

7. Extracting: Extracting a pattern of sequence from the whole string.

8. Cutting: Cutting DNA strands at specific points.

9. Ligating: Pasting DNA strands together.

10. Substituting: Substituting, inserting or deleting a sequence.

11. Detecting: Reading a DNA sequence from the solution.

DNA computing is done by generating a DNA sequence to represent a question and allowing all possible combinations of solutions to be generated by natural replication mechanism of DNA. After all possible solutions are generated in the form of different DNA strings, the class of solutions which needed to be eliminated are filtered by using the molecular biology tools mentioned above. After elimination, what is left over represents the class of solutions to the problem posed.

It should be noted that DNA computing is not suitable for all types of computing. The class of problems that can be solved by DNA computing effectively are problems that require massive parallelism and extensive number of trials. A good source for the type of problems suitable for DNA computing is given in [8].

Even though the speed of DNA replication itself is not very high, massive parallelism of DNA computing makes the overall data processing very efficient. The speed of DNA replication is 500 base pairs per second. Considering 2 distinct base possibilities for every base, this rate amounts to 1000 bits/second processing speed in terms of conventional computing terms. As soon as the replication of a base ends, the finished base starts being replicated as well. So the number of base pairs replicated increases exponentially. In conventional terms, this means increasing processing speed with every iteration. As an example, at the 30 th iteration the processing speed is $2>30 \times 1000=1000$ Gbits $/ \mathrm{sec}$.

The bases in a DNA are spaced at every 0.35 nanometers. According to this, DNA has a linear data density of 18 Mbits/inch. Extending the calculation to two dimensions, assuming a space of $1 \mathrm{~nm}$ between the pairs, data density becomes 1 million gigabits per square inch.

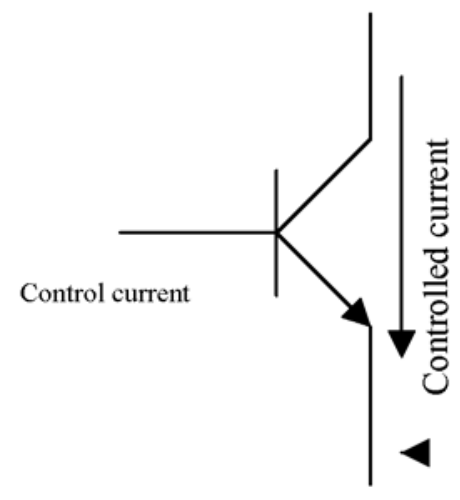

a) Bipolar transistor

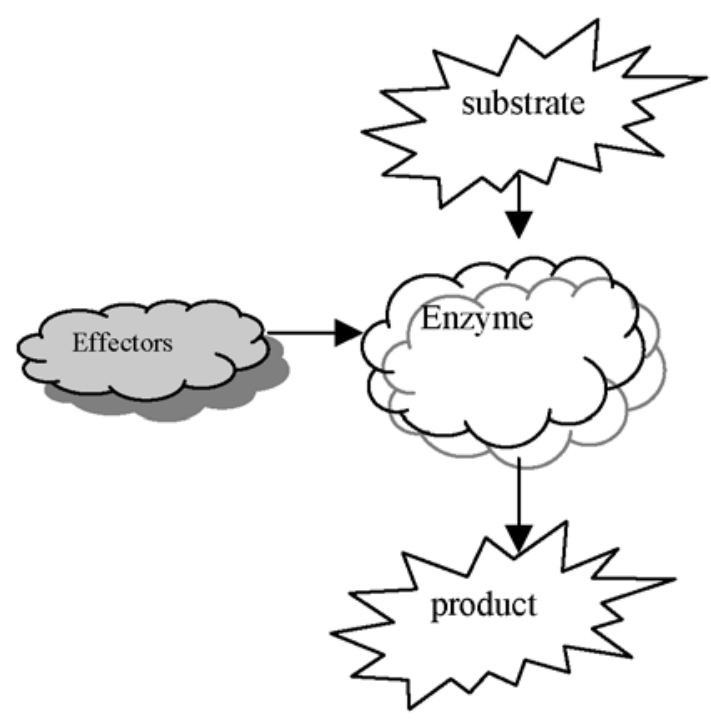

b) Enzymatic transistor

Fig. 1. In an enzymatic transistor effectors control the rate of conversion of substrate into product. 


\section{Whole Cell Computing}

Another form of biological computing is whole cell computing. In this approach, enzymes and cells are used to perform logic like functions. Unlike DNA computing, computing with whole cells and enzymes generates decision path similar to conventional computers [9].

A basic biological equivalent of a logic gate has been constructed successfully by using biological transistor [10]. In silicon-based computational logic, gates are constructed using electrical transistors which have base, emitter and collector terminals. The base current injected to the base terminal controls the current that flows from collector terminal to the emitter terminal. The control current injected to the base terminal is several orders of magnitude smaller than the controlled current of collector-emitter terminals.

In a biological transistor, there is no current involved. An enzyme, which is the biological equivalent of transistor, converts one kind of substrate into a different kind of product. The rate of conversion is controlled by effectors in Figure 1 [11]. An OR gate made from this type of enzymatic transistor is effected by two or more kinds of effectors. A logic gate of this kind is constructed by using biolumincense [10].

A cell called Pseudomonas putida (TVA8) converts Myristic acid into light in the presence of inducer toluene or trichloroethylene (TCE). In this example myristic acid is the input substance of the biological transistor, light is the output substance and toluene or TCE is the input trigger that controls the conversion process. In this case the generation of light is controlled by either one of these inducer effectors. The overall behavior of this setup acts like an OR

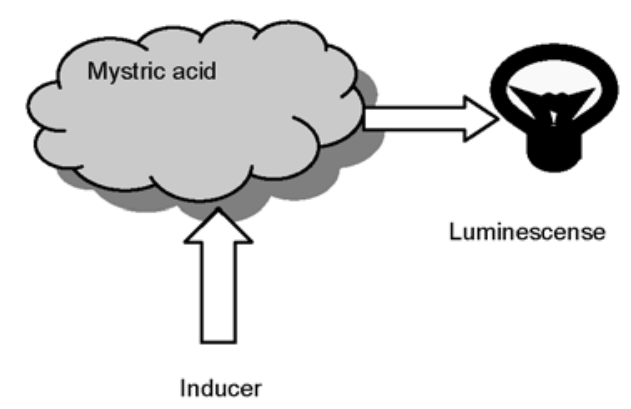

Fig. 2. Bioluminescence is controlled by the inducer. gate generating output (light) if either one of the inputs (TCE or toluene) is present.

In order to solve complicated problems one needs many logic gates to function together. Establishing interconnectivity between different biological logic gates can be done by employing mechanism of intercellular communication. An example of this type of communication is known as AHL and it is used for controlling population of the colony. Normally, every cell produces certain amount of AHL and releases it to the surrounding. When the concentration of the AHL reaches a level due to the increased number of cells, the cell production is supposed to be stopped. This sort of intercellular communication is the natural way cells communicate. A similar mechanism can be used for interconnectivity of cells to make up bigger system [12].

Contrary to expectations biological computing elements can be remarkably fast. A simple bacteria called Halobacterium halobium functions as a light driven proton pump and can be used to make a bistable red/green light switch which operates at 500 femtoseconds [5].

\subsection{Quantum Computing}

Another technology which promises extraordinary computational speed for special class of problems is quantum computing. Just like DNA computing, quantum computing is suitable for problems that require massive parallel processing.

In a quantum computer, information storage is called qubit, which represents the information in terms of quantum-mechanical states. Qubit is the equivalent of a bit in classical computers. Two cubits can represent the information in four different states, namely 00, 01, 10 and 11. The difference from the classical computer becomes apparent when it comes to processing information. In a classical computer one would need to enter these states to the digital system to get outputs one by one. In a quantum computer, all states enters the system simultaneously and all outputs are generated at the same time i.e. simultaneously. The outputs generated are in a superposed state and all available at the same time. The power of quantum computing becomes apparent when one considers large number of input cubits. Quantum gates can be 
connected back to back and turned on and off to implement complicated operations. The operation of a quantum computer is given in Figure 3. to indicate the operation of a quantum computer.

Currently, there are only few problems that can take advantage of the massive parallelism of quantum computers [13].

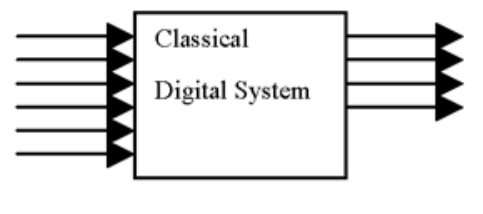

Only one input combination is applied at a given time

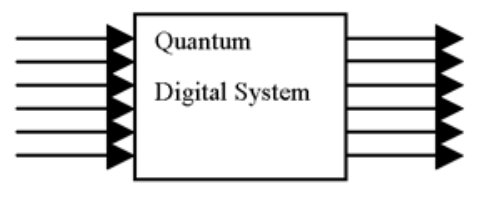

All input combinations are applied simultaneously. All outputs are available simultaneously in superposed state.

Fig. 3. Quantum computer gates.

Computing with quantum computers require initialization, processing and reading of cubits. There are currently more than one way of realizing cubits in hardware and this is likely to increase in the near future. Presently, the speed of a spin cubit gate made in silicon structure is in the order of $10^{-2} \mathrm{sec}$. and external operations dealing with cubits require $10^{-5} \mathrm{sec}$. Linear density of cubits in semiconductors is currently $65 \mathrm{~nm}$ [14]. Although the speed does not seem very impressive, considering the inherent parallelism of each cubit gate, the overall processing speed is likely to be very high.

\subsection{Reversible Computing}

Reversible computing receives its name from the fact that reversible gates work both ways; forward and backward. In other words, there are no dedicated input and output lines in a reversible gate. All lines, depending on the operation, can be either input or output lines. In addition to being reversible in direction of operation, the gates used in reversible computers are also thermodynamically reversible. Thermodynamic reversibility means that the energy lost in changing the state of the gate is regained when the gate is changed back to the original state. Only small amount of energy is lost during the cycle of going and coming back from a state. This type of energy reversibility reduces the power consumption of the device to minimum levels so that many gates can be crammed into a VLSI package since power consumption and dissipation is reasonable.

There are several designs made for reversible gate structures. One of the structures proposed by Merckle in 1993 is known as helical logic. In this design, the movement of single electron is controlled by control logic. The operation of the helical transistor can be explained as follows. An electron, which has two different paths to choose, is shown in Figure 4. The path of the electron is considered the data path. The

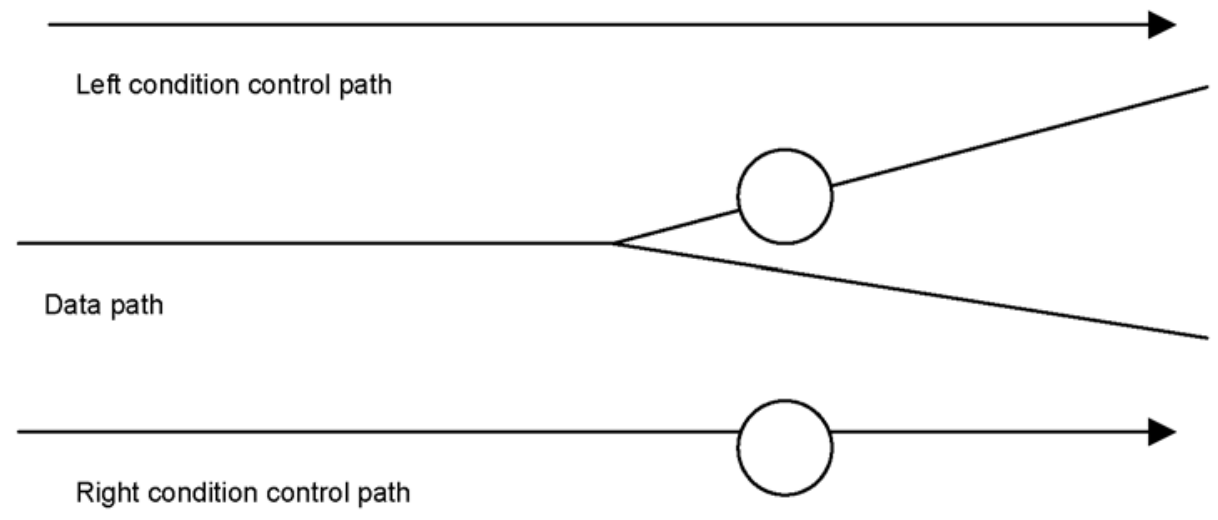

Fig. 4. Schematic of a two control path helical switch gate. 
movement of the electron, selection of right or left path, is controlled by the electrostatic forces that act on the electron. The paths on both sides of the data path are called control paths. The electron on the data path is forced to take the right path if we send an electron on the left control path simultaneously. The electrostatic force that acts on the data electron will force the electron to take data path on the right. Likewise, if we want the data electron to take the left path, we should send a synchronized electron on the right control path [15].

The name helical logic comes from the fact that the data and the control paths are actually rotating electric fields which are easy to produce and adequate to drive wide range of logic devices [15]. Being thermodynamically reversible, this type of circuit can be packed densely, can operate at gigahertz range frequency and consumes very little power. Expected density of the devices is in the order of $1 \mathrm{~nm}$ [16].

An advantage of this technology over the others is that, this technology is based on variation of silicon technology we are quite familiar with. There is still much needs to be done before making this technology mainstream, but it seems one of the promising technologies on the drawing board.

\subsection{Optical Computing}

Another promising technology of computation is optical computing. Optical computing has been around us for quite some time and used for experimental and research setups. So far it has not been accepted as mainstream technology because of problems with input power requirements of optical logic circuits and the lack of miniaturization. Most optical setups required high power laser beam and it is not possible to reduce the size to make it practical.

However, recent developments in optical computing have made optical computing a serious contender in alternative computing technology arena. The new approach utilizes optical computing not to replace but to enhance the current silicon processing technology.

Current computer architecture makes use of internal buses to connect different gates implemented on the silicon hardware. Implementation of the buses on VLSI hardware occupies considerable real estate space on the IC chip. Since the data can be transferred only in one direction at any given instant, the operation of the buses also stifles the speed of operation. Utilizing optical conduits as internal buses for transferring information in the form of light pulses present many advantages over the conventional bus for transferring information. By using different color pulses, it is possible to carry many sets of data simultaneously. The technique called wavelength division multiplexing can be used very effectively to increase the overall performance of the exiting processors.

Together with the new optical switching materials that operate at 60 Gigahertz and wavelength division multiplexing implemented inside CPU, we can expect computers with performance of 1017 operations per second [17].

A new type low energy optical switch utilizing this hybrid technology is called symmetric self-optical effect device. S-SEED works as an electronically controlled reflective switch. By controlling a gate voltage, reflective properties of the device can be changed. A schematic of an S-SEED device is shown in Figure 5 [18].

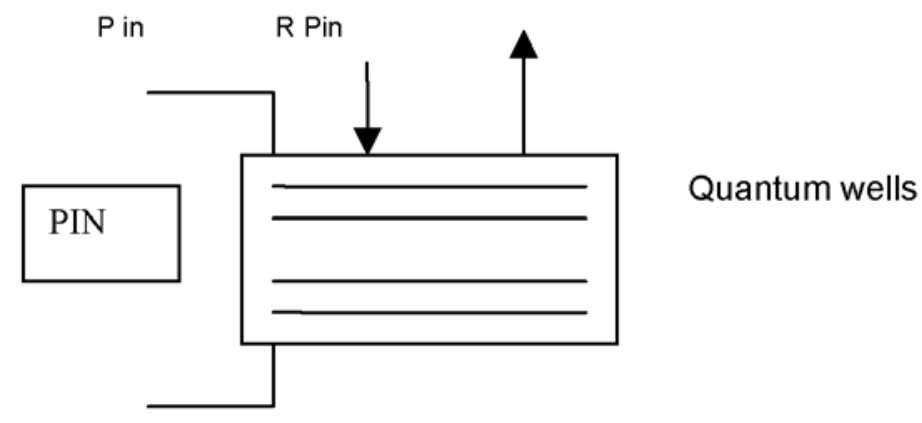

Fig. 5. Structure of S-SEED device. Absorption of the input beam Pin is modulated by voltage applied to PIN input of the device. RPin is the reflected beam which is modulated. 


\begin{tabular}{|c|c|c|c|}
\hline Computing Technology & \begin{tabular}{|} 
Switching \\
speed or \\
overall \\
processing \\
speed
\end{tabular} & $\begin{array}{l}\text { Linear } \\
\text { density }\end{array}$ & Class of problems suitable \\
\hline "DNA computing & $\begin{array}{c}1000 \\
\text { gbits/sec. }\end{array}$ & $\begin{array}{c}0.35 \\
\text { nanometers }\end{array}$ & $\begin{array}{l}\text { Suitable for NP class problems which require } \\
\text { many iterations and selection from many } \\
\text { possible solutions. }\end{array}$ \\
\hline Whole cell computing & $\begin{array}{c}500 \\
\text { femtosec. }\end{array}$ & $\begin{array}{l}\sim 1 \text { micro } \\
\text { meter }\end{array}$ & Classical computing problems. \\
\hline Quantum computing & 10 millisec. & $\begin{array}{c}65 \\
\text { nanometers }\end{array}$ & $\begin{array}{l}\text { Suitable for NP class problems which require } \\
\text { many iterations and selection from many } \\
\text { possible solutions. }\end{array}$ \\
\hline Reversible computing & 1 gigahertz & 1 nanometer & Classical computing problems \\
\hline Optical computing & 60 gigahertz & $\begin{array}{c}0.35 \\
\text { micrometer }\end{array}$ & Classical computing problems \\
\hline
\end{tabular}

Table 1. Comparison of existing alternative computing technologies.

S-SEED device can be manufactured in a hybrid chip form integrated with traditional switching gates. SEED device offers the possibility of using optical signals as input and output signals to traditional VLSI chips. SEED integrated hybrid chip technology is called smart pixel and can be manufactured with traditional manufacturing technology. Through this method it seems that the pin out limitation of the VLSI chips can be tackled and power consumption can be reduced.

\section{Comparison of Alternative Computing Technologies}

The Table 1 above lists the potentially promising technologies we expect to see in the near future.

\section{Conclusions}

In this paper we have discussed several technologies which seem promising as alternative technologies for high performance information processing applications. All technologies are at their infancy and much remains to be done in order to make them mainstream technology. The technologies are suitable for different classes of problems. DNA computing and Quantum computing may provide quick and easy solutions to problems which may be very difficult for classical sequential computers. Some technologies are suitable for implementation as hybrid technology to enhance the performance of the existing silicon computers. Which technology becomes dominant is not necessarily dependant on the technical ability of the technology, but also on the political aspects of promoting the technology. It is also possible that none of the technologies becomes dominant and each technology is limited to solving only very specific problems.

Although the figures for processing speed and density of each technology are given, these figures alone do not represent the power of processing. Density figures indicate the current state of the art, but they are likely to be revised as the technology matures.

Quantum computing and optical computing are the two categories that make use of the existing silicon technology. In this respect, these two technologies have a head start and can rapidly unfold hybrid designs which improve the performance of existing computing performance. 


\section{References}

[1] C. GWYN, Extreme ultraviolet technology for next generation IC's, Intel Corporation, http: //www.intel.com/research/silicon /euv_mpf_101100.pdf

[2] F. L. CARTER, R. E. SiatKowski, AND H. WohltJEN, Molecular Electronic Devices, North-Holland, Amsterdam, 1988.

[3] K. E. DREXLER, Nanosystems: Molecular Machinery, Manufacturing, and Computation, Wiley Interscience, 1992.

[4] R. C. MERKLE, Reversible Electronic Logic Using Switches, Nanotechnology, 4 (1993), 21-40.

[5] C. R. Lowe, Nanobiotechnology: The fabrication and Applications of Chemical and Biological Nanostructures, Current Opinion in Structural Biology 10 (2000), pp. 428-434.

[6] D. GHOSH, High throughput and global approaches to gene expression, Comb. Chem. High Throughput Screen 3 (2000), pp. 411-420.

[7] G. S. WILsON, AND Y. B. Hu, Enzyme-based biosensors for in vivo measurements, Chem. Rev. 100 (2000), pp. 2693-2704.

[8] L. KARI, DNA computing in vitro and in vivo, $F u$ ture Generation Computer Systems 17 (2001), pp. 823-834.

[9] L. BousSE, Whole cell biosensors, Sensor Actuat. 34 (1996)B, pp. 270-275.

[10] M.L. SimPSON, Bioluminescent bioreporter integrated circuits (BBICs), in Smart Structures and Materials Smart Electronics and MEMS (1998), pp. 202-212.

[11] M. Hiratsuka, T. AOKI And T. Higuchi, Enzyme transistor circuits for reaction-diffusion computing, IEEE Transactions on Circuits and Systems I, Vol. 46, No. 2, (1999), pp. 294-303.

[12] M. L. SIMPSON, Whole-cell Biocomputing, Trends in Biotechnology 19 (2001), pp. 317-323.

[13] P. W. SHOR, Algorithms for quantum computation: Discrete logarithms and factoring, Proc. 35nd Annual Symposium on Foundations of Computer Science (Shafi Goldwasser, ed.), IEEE Computer Society Press (1994), pp. 124-134.

[14] V. Privman, D. Mozyrsky, I. Vagner, Design of Gates for Quantum Computation: the Three-Spin XOR in Terms of Two-Spin Interactions, Int. J. Modern Phys. B(1998) 12, pp. 591-600.

[15] K.E DrEXLER, Helical Logic, Nanotechnology, 1996, pp. 325-339

[16] J. LEWIS ED. Nanocon proceedings, (1989) pp. 2.

[17] H. ABDELDAYEM, Recent advances in photonic devices for optical computing, NASA publication. http://science.nasa.gov/headlines /images/nanosecond/thepaper.pdf
[18] B.S. WHERETT, Materials for Optical Computing, Synthetic Metals 17 (1996), pp. 3-9.

Received: July, 2002

Revised: May, 2003

Accepted: June, 2003

Contact address:

Tarik Ozkul

American University of Sharjah Computer Engineering Division
Sharjah

Sharjah

e-mail: tozkul@ausharjah.edu

TARIK OZKUL is a professor at the American University of Sharjah. His research field is computer architecture and computer applications to industry. 